\title{
Disputas por el sentido y el acceso a la práctica de esquí en Bariloche (1999-2013)
}

\section{Disputes over the sense and access to the practice of skiing in Bariloche (1999-2013)}

\section{Leonardo Daniel Mársico*}

\section{* Profesor de Educación Física (Universidad Nacional del Co- mahue, Argentina). Magíster en Ciencias Sociales y Humanidades (Universidad Nacional de Quil- mes). Referente pedagógico de programas de la Dirección de Educación Física y Artística de Río Negro. Profesor adjunto y miem- bro del grupo de investigación «Instituciones, prácticas educati- vas, corporales y culturales» (Uni- versidad Nacional del Comahue). \\ •leomarsico@gmail.com \\ http://orcid.org/0000-0002- \\ 0224-5330}

RECIBIDO: 5.9.2021

ACEPTADO: 8.9.2021

\begin{abstract}
Resumen
La nieve y el esquí son íconos de Bariloche y están presentes en un imaginario argentino que considera que todas y todos sus habitantes pueden acceder a la práctica del esquí. Sin embargo, el devenir histórico, con un pasado de opresiones, inversiones discrecionales y centralismo nacional, acentuados con la participación protagonista de Parques Nacionales a partir de 1934, fue constituyendo disposiciones materiales y simbólicas que excluyeron de la práctica del esquí a la mayor parte de la población barilochense. La evidencia relevada en este estudio indica que, si bien la práctica del esquí en Bariloche se asentó principalmente con una dimensión deportiva y recreativa para un sector social alto o medio-alto, con el transcurrir de las décadas surgieron propuestas que promovieron el acceso de sectores sociales más amplios, lo que ha favorecido la apropiación de prácticas, sentidos y entornos construidos en la región.
\end{abstract}

Palabras clave: deporte, historia, actividad de tiempo libre, Argentina. 


\section{Abstract}

Snow and skiing are icons of Bariloche and are present in an Argentine imaginary that considers that all its inhabitants have access to the practice of skiing. However, the historical evolution with a past of oppressions, discretionary investments, and national centralism, accentuated by the leading participation of National Parks as from 1934, comprised material and symbolic dispositions which excluded most of the local population from skiing. The evidence gathered in this study indicates that although the practice of skiing in Bariloche was established mainly for a high or medium-high social sector, over time, many proposals emerged to promote access to skiing for broader social sectors, encouraging the appropriation of practices, meanings, and the natural surroundings from a local territory perception.

Keywords: sport, history, leisure time activities, Argentina.

\section{Introducción}

Bariloche, localizada en la Patagonia argentina, es una ciudad icónica por estar vinculada estrechamente a la nieve y la práctica del esquí, condicionada por su historia y geografía (Chiocconi, 2015; Chiocconi et al., 2011; Fuentes y Núñez, 2007; Arko, 2006; Bachmann, 2000). Esto se debe en parte a la sedimentación de un imaginario colectivo que se fue constituyendo mediante las prácticas vinculadas al turismo invernal que se promueven en medios masivos de comunicación y que se asocian, por nombrar algunas, a viajes de recién casados y al turismo estudiantil (Municipalidad de San Carlos de Bariloche [MSCB], 2021). Este imaginario sostiene la idea que en Bariloche «todas y todos esquían», lo cual se contrapone con la realidad, en la que solo una proporción reducida de residentes accede a la práctica del esquí.

Situada dentro del Parque Nacional Nahuel Huapi, Bariloche posee el centro de esquí más grande de Sudamérica, ubicado en el cerro Catedral, con gran cantidad de pistas y medios de elevación. La enorme mayoría de las personas que esquían son turistas, mientras que menos del 7\% de los barilochenses (aproximadamente unos 9000 habitantes de una población de más de 133 500) ${ }^{1}$ acceden a esquiar en el cerro Catedral (Catedral Alta Patagonia S.A. [CAPSA], 2018). Según algunos estudios de la actividad económica y de la planificación de Bariloche (Ros \& Assoc., 1998; MSCB, 2008, 2011 y 2015), el turismo es el principal recurso económico en una ciudad que históricamente ha poseído grandes deficiencias sociales, culturales y económicas, y donde se permite 
que el mercado regule la distribución del espacio y su acceso (Medina, 2017; Matossian, 2015; Núñez, 2014; Núñez y Vejsbjerg, 2010) (figura 1).

El esquí es para el turismo una propuesta seductora, una imagen de publicidad para los visitantes que viajan hacia Bariloche, mientras que, para la población residente de los sectores medios el acceso es eventual y escaso o nulo para los sectores populares. En este trabajo se analizan las acciones y los proyectos destinados a revertir esta tendencia que fueron surgiendo en las últimas décadas. En este recorrido, distintas propuestas de enseñanza del esquí se presentaron como la única posibilidad de acceso a esta práctica para sectores vulnerados de la sociedad, y fueron aumentando en número y solidez hasta lograr su institucionalización en la escuela primaria. Historia, prácticas y sentidos se entrecruzan mostrando un recorrido por el cual el esquí va siendo tensionado por personas, instituciones, voluntades, intereses y recursos, en un proceso local que cuestiona el sentido de la práctica.

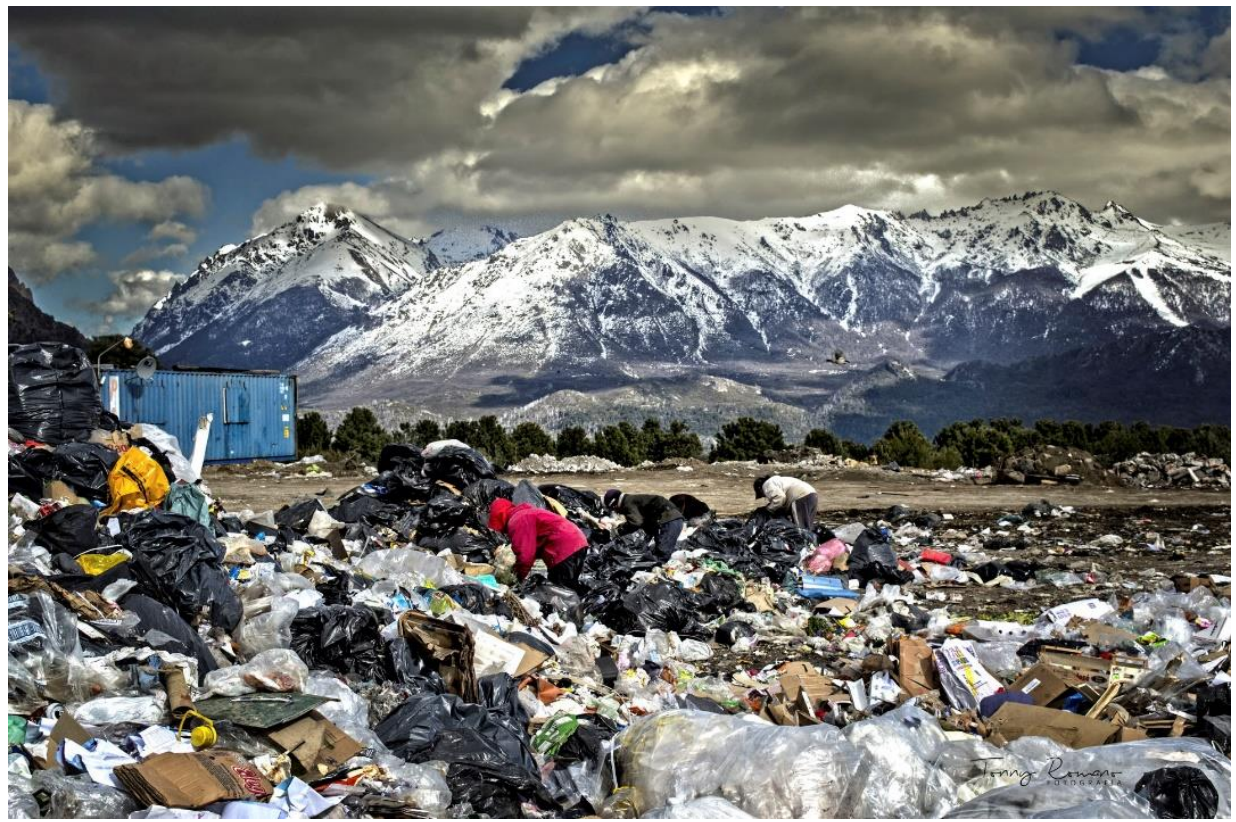

Figura 1. Cerro Catedral con el centro de esquí sobre la esquina superior derecha, visto desde el vertedero ubicado al sur de la ciudad de Bariloche. Se observan personas recolectando basura reciclable. Fuente: Desde lejos no se ve, fotografía reproducida con permiso de su autor, Tonny Romano (tonnyromano.com). 


\section{El esquí en la región patagónica}

\section{Conformación de la identidad local: \\ el sentido social de la práctica del esquí en Bariloche}

Ya desde los pueblos originarios, antes de la fundación de la Colonia Agrícola Ganadera Nahuel Huapi, en 1902, hubo una integración en las comunidades de la región patagónica a un lado y otro de los Andes. Estos lazos comenzaron a obstaculizarse a partir de la llegada de migrantes, posterior al proceso de campañas militares genocidas para la expansión territorial del Estado argentino hacia la Patagonia, llevado adelante por Julio A. Roca (1878-1885).

Los habitantes del poblado que en 1903 devendría en San Carlos de Bariloche (situado en un territorio que dependía directamente del Poder Ejecutivo Nacional) sentían abandono y exclusión respecto de las autoridades centrales, dado que estas no tenían en cuenta sus propuestas ni demandas, lo que afectaba la identidad regional (Arias Bucciarelli, 2009).

Los proyectos de integración local ligados al ambiente natural y pensados desde Bariloche intentaron redireccionar estrategias económicas mediante alternativas que no fueron tenidas en cuenta por los gobiernos nacionales (Navarro Floria y Vejsbjerg, 2009). Son ejemplo de esto las propuestas de Bailey Willis primero y Emilio Frey después, para la creación de un parque nacional (en línea con el destino inicial propuesto por Francisco Moreno).

En las primeras dos décadas del siglo XX, entre los sectores porteños mejor acomodados, que podían acceder a la región para recorrerla y disfrutarla, se producía un fenómeno de promoción que otros desarrollarían turísticamente más adelante, mediante publicaciones periodísticas, de fomento y, ocasionalmente, producciones artísticas (Aarón de Anchorena, Ada María Elflein, Emilio Morales, con libros, fotos, postales, etcétera).

Las decisiones políticas nacionales serían determinantes para una transformación local orientada hacia el desarrollo turístico. En 1922 fue creado el Parque Nacional del Sud, que no generó cambios sustanciales en la dinámica regional. En cambio, en 1934, el proyecto para reconstituir los Parques Nacionales fue un hito que cambiaría el sentido de la región en términos políticos, presupuestarios y simbólicos dentro del territorio nacional. Esta propuesta se personalizó centralmente en Exequiel Bustillo cuando se crearon la Dirección de Parques Nacionales y el Parque Nacional Nahuel Huapi (junto con el Parque Nacional Iguazú), que dirigió hasta 1944.

En este tiempo comenzó a sistematizarse un proyecto social, político y económico en la región, elaborado en Buenos Aires y estructurado mediante organismos y 
recursos del Estado Nacional, del cual el esquí era una parte simbólica y práctica significativa. Junto con la culminación de la obra del ferrocarril Buenos Aires-Bariloche, en diciembre de 1934, la región vivió un proceso de transformación intenso, y un factor que lo aceleró fue la disposición efectiva de recursos a través de Bustillo. En el marco del gobierno conservador, proveniente de la aristocracia porteña, se potenció una política de Estado que miraba hacia Buenos Aires como visión cultural hegemónica y nacional, sin respetar las subjetividades regionales (Navarro Floria y Vejsbjerg, 2009). Esta mirada centralista y aristocrática comenzó a disputarse el sentido social de la región para la práctica del esquí.

El uso del pseudónimo la Suiza argentina para referirse a Bariloche había aparecido varios años antes. Su utilización y ejecución práctica se materializó efectivamente de la mano de Bustillo como uno de los mayores procesos de crecimiento local, que autoproclamó posteriormente en su libro, El despertar de Bariloche (Bustillo, 1968). Esta nueva forma de concebir la ciudad implicaba no solo lo arquitectónico, sino un proyecto de Estado que intervendría en sus modos de producción y su vinculación comercial y social. En cuanto a la presencia de los modos de conducción del Parque Nacional, Bustillo fue muy claro en la discrecionalidad con la que se manejaba al frente de la institución. Ejemplo de ello es el uso que hacía de la tierra en beneficio propio y de amistades, intentando que todas aquellas personas que fueran importantes para él adquirieran un «pequeño lote de tierra para levantar algún día su residencia veraniega» (Navarro Floria, 2008, p. 8).

En los primeros períodos presidenciales de Perón (1944-1955), se modificaron en parte la estructura y el sentido de los parques nacionales. La proyección elitista propuesta por Bustillo se dejó de lado para darle un cariz más popular, al que podrían acceder contingentes mayores.

Estas prácticas, promovidas por el Estado Nacional, fueron acciones enmarcadas en el turismo social (Núñez y Vejsbjerg, 2010), pero no se sentían como propias en el espacio local barilochense. A pesar de que el Club Andino Bariloche (primer club de montaña, fundado en 1931) interactuó con el Gobierno Nacional para la construcción de refugios de montaña y para la promoción deportiva local, en documentos de su archivo se encuentran miradas antiperonistas (Núñez, 2014).

Completado el proceso de provincialización del territorio nacional y luego del gobierno de facto de la autoproclamada Revolución Libertadora (1955-1958), el Estado Nacional diferenció sus intereses en la región; promovió inversiones vinculadas al desarrollo energético y centradas en el alto valle del río Negro y en la zona de Viedma (haciendo hincapié en los centros mineros y las superficies irrigadas para cultivo). En estos casos, el desarrollo turístico en la región no aparece siquiera reflejado como actividad económica para la inversión (Núñez, 2014).

En un análisis más preciso, la urbanización de Bariloche siempre estuvo antecedida por la demanda del mercado inmobiliario privado y especulativo, antes que por 
planeamientos estratégicos que dieran un sentido algo unificado a su población (Medina, 2017; Matossian, 2015; Guevara y Núñez, 2014; Fuentes y Núñez, 2007). «Los intereses privados avanzaron en un ordenamiento espacial que fue diferenciando cada vez más al Parque Nacional de la ciudad en crecimiento. El paisaje fue cada vez más ajeno a sus habitantes» (Núñez, 2014, p. 8).

A pesar de la falta de inversión y planificación vinculada al turismo, este funcionó como motor económico. Los registros indican que el número de turistas anuales se triplicó —pasó de 144.000 en 1966 a 423.000 en 1975—, acompañado de un aumento poblacional vinculado a la demanda de infraestructura (Fuentes y Núñez, 2008).

\section{Recorridos de la práctica del esquí}

\section{Disputas por el sentido de esquiar}

Los primeros registros, que datan del período 1890-1930, evidencian el desarrollo y los diferentes sentidos de las prácticas de esquí, equivalentes a su genealogía a nivel global. Las primeras manifestaciones de la utilización de esquíes en el marco de la Colonia Agrícola-Ganadera Nahuel Huapi remiten a su empleo como transporte, para llegar de algún lugar a otro (Chiocconi et al., 2011). La información indica que en ningún momento hubo referencias a las sensaciones de los esquiadores u otro sentido de la práctica.

En la década de 1930, con el Club Andino como institución primordial, aparece un sentido recreativo en el desarrollo de la práctica del esquí realizada de manera grupal y familiar. ${ }^{2}$ Difundida por algunas personalidades locales, esa práctica se muestra vinculada al tiempo libre y al goce. Es de destacar que quienes conformaban el Club Andino y quienes disponían de tiempo para prácticas de esquí eran justamente las y los barilochenses que estaban en mejor posición socioeconómica que el resto - «comerciantes, empresarios, profesionales y empleados»- y que de las actividades no participaba «gran parte de la población de la zona, como los trabajadores más humildes, los chilenos pobres y los indígenas» (Chiocconi et al., 2011, p. 234). Pese a que la visibilización del esquí aumentó durante la Década Infame (1930-1940), el fútbol, el ciclismo y el tiro fueron las prácticas deportivas que adquirieron mayor cantidad de participantes y creación de instituciones (Chiappe y Méndez, 2021).

Entre las dos perspectivas de desarrollo que proyectaba la ciudad de Bariloche, se destacó una tensión análoga que pugnaba en la definición de los modos y los fines de la práctica del esquí. Las definiciones tomadas en Bariloche y las proyectadas desde 
Buenos Aires se encarnaron en dos personas con sentido gravitante: Otto Meiling ${ }^{3}$ y Hans Nöbl ${ }^{4}$ (Chiocconi et al., 2011; Navarro Floria, 2008; Bachmann, 2000). Ellos personificaron dos modos paradigmáticos de vivir la práctica del esquí, mediante distintas vinculaciones posibles con el ambiente de montaña y con los medios de producción locales. Esta diferenciación podría describirse brevemente en dos hipótesis opuestas que se disputarían el destino de Bariloche: vivir en las montañas con el esquí (tendiente al pensamiento de Meiling) o vivir del esquí y las montañas (tendiente a la propuesta de Nöbl).

La tensión entre sus instituciones de pertenencia fue evidente en la disputa por la enseñanza del esquí. La Escuela de Esquí Argentina del Club Andino era preferida por el público local, mientras que la otra escuela de esquí, formada por instructores europeos en el cerro Catedral de Parques Nacionales, tendía a acaparar a la élite visitante (Navarro Floria, 2008; Bachmann, 2000). En ese contexto, algunos relatos dejan en evidencia que las y los niños de Bariloche no iban a esquiar, a menos que contaran con los recursos económicos para hacerlo (Bachmann, 2000).

El modelo proyectado por la aristocracia porteña fue ganando preponderancia a través de los recursos de Parques Nacionales (1934-1944) y dirigiéndose a los sectores sociales medio-altos y altos para la explotación turística de las dimensiones recreativas y deportivas del esquí. ${ }^{5}$ El enclave agrícola, ganadero y maderero vinculado a los poblados de la región se fue transformando en una ciudad turística que intentó mostrar características europeas, orientada al disfrute de las elites, con la nieve y el esquí como íconos indelebles. En contraposición a esta tendencia, la disposición excluyente del esquí fue encontrando en su camino quiebres y cambios de dirección que habilitaron, promovieron y sostuvieron otras posibilidades de acceso.

\section{Intentos de incorporación de sectores excluidos a la práctica del esquí}

Cobra notoriedad en el ámbito del esquí Catalina Reynal, quien venía a esquiar a Bariloche y poseía vínculos en los estratos sociales altos de Buenos Aires. Reynal creó una escuela para niños que no podían acceder al esquí. Su idea surgió al ver a un grupo de hijos de empleados del centro invernal jugando con esquíes rudimentarios en las gró como montañista tanto por sus logros como por su acción y vida en la montaña.

4 Hans Nöbl (o Nôbl), austríaco contratado por Parques Nacionales para el desarrollo de un centro de esquí en la región, al final de la década de 1930.

5 «Promoción turística 1940», s.d. 
pendientes fuera de las pistas. Reynal sostuvo su escuela de esquí entre 1943 y 1953 mediante un acuerdo específico con Parques Nacionales, y en ella mantuvo la participación de solo unos 30 niños y jóvenes (Bachmann, 2000). Aparentemente, la mayoría de los niños que asistían a esa escuela se repetían año a año. Por entonces, aquellas prácticas deportivas que habían tomado notoriedad en la década de 1930 comenzaron a perder protagonismo. Durante los períodos peronistas de gobierno, el básquet y el boxeo mostraron un crecimiento más acelerado, mientras que el fútbol no parece haber variado su tendencia de desarrollo (Chiappe y Méndez, 2021).

Durante los últimos años del segundo gobierno peronista, la propuesta se trasladó al Club Andino. La creación del Ski Club en 1966, a partir de la separación de varios de los miembros del Club Andino, se produjo, en parte, por la dificultad de continuar el programa que Reynal seguía sustentando y por diferencias en la elección del entrenador a cargo. Reynal aportaría fondos al Ski Club para el pago de un instructor de esquí destinado al entrenamiento deportivo del equipo. En este hito se exteriorizan dos aspectos. El primero es que la propuesta original dejó de tener una valoración y una intención orientadas al acceso y el desarrollo, para consolidarse como una versión deportivizada de la práctica, destinada a los que ya podían realizarla. El segundo aspecto es que había disputas locales por la administración de los recursos que Reynal aportaba (suficientes como para que, en 1948, un grupo de sus alumnos participaran en los V Juegos Olímpicos de Invierno de Sankt-Moritz).

Esa intención de acercar a niñas y niños de Bariloche a las prácticas de esquí, dada su condición social desfavorecida y la imposibilidad material de hacerlo por su cuenta, se vuelve a relevar recién a principios de la década de 1980, en la propuesta que realizó María Bachmann junto con Julio Fernández, ambos instructores locales. Acompañados de varios instructores en formación (que aprovechaban a realizar sus prácticas profesionales en ese espacio), se organizaron para dar clases de esquí a unas 80 alumnas y alumnos durante septiembre y octubre, meses de escasa afluencia de turistas. Bachmann, que podía acceder a las escuelas públicas del Consejo Provincial de Educación (CPE) con mayor facilidad por su condición de profesora de Educación Física, coordinaba la selección de las o los dos mejores alumnos que, además, se destacaran en Educación Física.

En términos de reproducción de diferencias sociales, en parte con una mirada meritocrática, se aprecia que quienes podían participar en esta actividad de esquí totalmente gratuita eran aquellas o aquellos alumnos que gozaban de cierta valoración de sus profesores. Quienes carecían de este mérito también se perdían la chance de esquiar.

Esta propuesta voluntariosa y restringida tomó carácter público y en 1984 impulsó la ordenanza municipal promovida por Néstor Cejas, que creó la Escuela Municipal de Esquí (ordenanza 102-C-84, MSCB, 1984) (gráfico 1). Se dispuso como propósito 
el acceso de niñas y niños que por su condición socioeconómica no podían esquiar, en un marco de participación y disfrute de la actividad, de formación integral y vinculado a su permanencia en el sistema educativo. El plan era ambicioso, mucho más amplio; incluía más de cinco instituciones colaboradoras ${ }^{6}$ para llevar a 175 alumnas y alumnos. Con altibajos a través de los años, dados por las relaciones cambiantes entre las diversas instituciones participantes (Estado y entes privados), este plan continuó articulando formas de difundir la práctica con sectores sociales desfavorecidos. Entre 1996 y 1999 la Municipalidad reelaboró la propuesta según esta misma norma. La elección del esquí de fondo (modalidad nórdica parecida a deslizar caminando) antes que el esquí alpino (modalidad de descenso que precisa de medios de elevación) convino porque, técnicamente, se realiza en un medio mucho más plano y, económicamente, no implicaba costos para los pases de esquí. A su vez, en esos tiempos las pistas del cerro Otto recibían mayor cantidad de nieve, y llegar a ese cerro implicaba un desplazamiento menor que el necesario para llegar al cerro Catedral.

A partir de 1999, en la Escuela n. ${ }^{\circ} 311$, situada en la periferia de Bariloche, se produjo un proceso de apropiación y resignificación pedagógica de esta propuesta de esquí. Entre el CPE, el Municipio y el Club Andino se llevó a cabo la primera experiencia de lo que, de manera tortuosa pero ininterrumpida, continuaría en un proceso de incorporación creciente de la práctica del esquí en escuelas públicas (gráfico 1). Con muchísimas variantes, tensiones, cambios de instituciones y personas, la propuesta, que coloquialmente se conoce como Esquí Escolar, se tornó transversal a toda la ciudad: a partir de 2013 , todas y todos los alumnos de $5 .^{\circ}$ grado de escuelas públicas, gratuitas y obligatorias llegarían a esquiar. Desde entonces (y hasta las restricciones sanitarias por la pandemia de covid-19), este programa de acceso y desarrollo pedagógico de la práctica del esquí, que pasó al ámbito del Ministerio de Educación de Río Negro en 2015, se sostuvo continuamente. tores de Esquí (hoy ISES-AADIDES); Centro Nacional de Educación Física n. 8 (Ayekan Ruca), Dirección Provincial de Deportes y Juventud, y las empresas del centro de esquí Robles S. A. y Lado Bueno. 


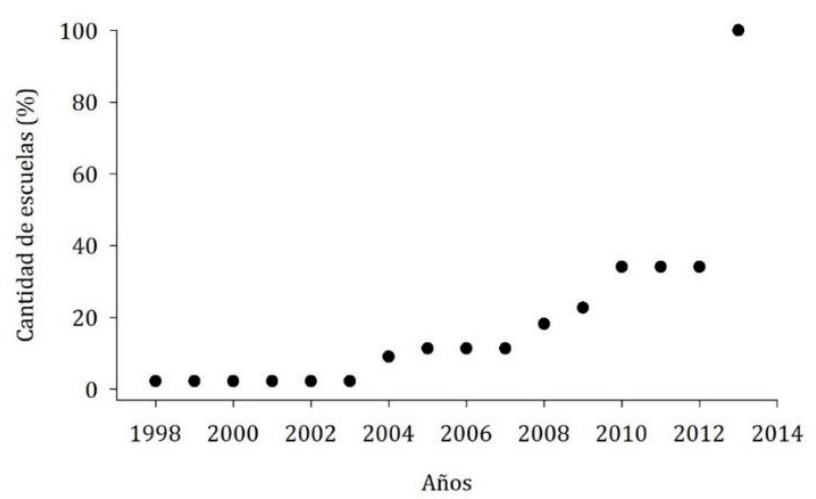

Gráfico 1. Incorporación de escuelas públicas a partir de su sistematización en Esquí Escolar en el marco de la educación formal. El 100\% de las escuelas representa a 44 instituciones de la educación primaria común del Consejo Zonal de Bariloche y congrega cada año alrededor de 1500 alumnos y alumnas, que se renuevan anualmente. Fuente: Mársico (2020).

\section{Contexto de institucionalización simbólica del esquí como práctica deportiva y turística}

La práctica deportiva del esquí fue sumando presencia simbólica y material con un alto desarrollo de sus instituciones en América y Europa, a la vez que el turismo comenzaba a hacerse presente para ciertos sectores sociales con poder económico alto, sobre todo después de la Segunda Guerra Mundial. En simultáneo, los sports —en términos generales - se desarrollaban en Occidente como fenómeno social, se potenciaban sus instituciones y se les daba lugar a sus prácticas en las escuelas (Scharagrodsky, 2011; Kirk, 2010; Aisenstein y Scharagrodsky, 2006; entre otros).

Se aceleró un proceso de institucionalización de organizaciones relacionadas con el esquí que ponderaba la dimensión deportiva de la práctica y que acrecentaba su imagen como propaganda para el turismo. Para dimensionar su importancia en las prácticas de esquí en la región patagónica puede mencionarse la creación de tres clubes (CUBA, CAB y CAS), con sus sedes centrales y refugios en la montaña, además de la federación que las agrupaba (FASA) para participar en otras uniones y asociaciones, todo en un territorio que aproximadamente tenía 150.000 habitantes ${ }^{7}$ pero implicaba a unos pocos (figura 2). La magnitud de tal impulso no podría haberse dado sin un contexto nacional 
e internacional relativamente alineado, favorable a este proceso de deportivización (como institución competitiva, normada, estandarizada y federada).

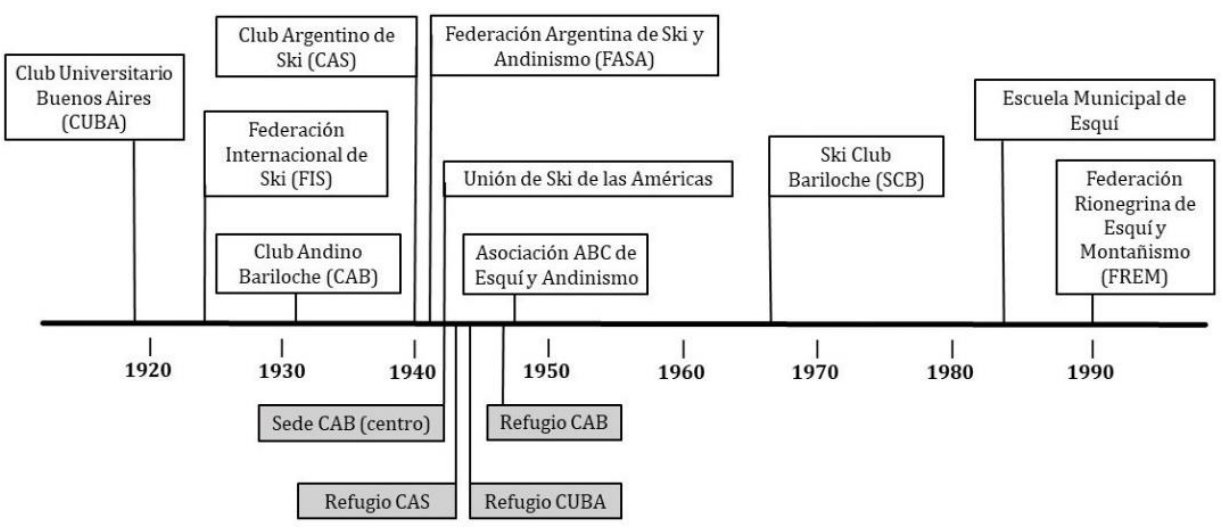

Figura 2. Línea temporal que indica la creación de organizaciones específicas de esquí vinculadas a Bariloche (cuadros blancos) y la inauguración de sus edificios locales (cuadros grises).

Fuente: Mársico (2020).

Este impulso del esquí, dado por sus prácticas vinculadas a la explotación comercial y los logros deportivos, fue aportando a la configuración de una imagen y un sentido distantes de lo manifestado antes de la década de 1940, que no parece haber cambiado significativamente hasta la actualidad.

Este fenómeno, orientado a sectores sociales altos y medio-altos, tuvo apenas una variación transitoria entre 1950 y 1970 de la mano de la función social de los gremios y el fomento al turismo social: «Bariloche será también un lugar de turismo para la clase media y los sectores populares» (Fuentes y Núñez, 2007, p. 25). En este período hubo un proceso de definición popular de los derechos de las personas, que en Bariloche no alcanzó a traducirse en un acceso real de sus habitantes a la naturaleza que los rodeaba, la cual «fue tornándose progresivamente ajena, y más ajena todavía a los sectores más vulnerables» (Núñez y Vejsbjerg, 2010, p. 943).

El sentido que fue adquiriendo el esquí puede enmarcarse en la distinción propia de la práctica en la que fue consolidándose y en el hecho de ser valorado y definido por los mismos sectores sociales que lo practicaban. El redireccionamiento del turismo a los sectores populares no alcanzó a afectar puntualmente el marco de explotación del recurso esquí. Los turistas que accedían a Bariloche aumentaron y variaron su origen, a la 
vez que se adaptó el formato de mercado que le daba sostén, mas no se modificó la accesibilidad intrínseca de la ciudad a su entorno ni, específicamente, el acceso a las prácticas de esquí.

\section{Algunas conclusiones}

\section{¿Quiénes pueden esquiar? \\ Acceso, costos, deporte y bien común}

La práctica masiva del esquí en la ciudad de Bariloche puede verse como una propuesta problemática, diferente y desafiante. En la región del noroeste de la Patagonia, en la cual lo constante es una gran oferta de turismo poco accesible para sus habitantes por sus altos costos, la realización del esquí alpino —una de sus prácticas más onerosas y distintivas, en términos de Bourdieu (1979/2002) - como algo masivo, inclusivo y popular para todauna franja social normalmente excluida presenta dificultades de índole simbólica.

Se puede apreciar que la preponderancia de la dimensión deportiva en el esquí y su instrumentación para la difusión turística tiene un sesgo histórico que la condiciona como práctica propia, y por ende distinguida, de sectores sociales con altos recursos económicos. Si el desarrollo de los deportes de costo moderado o alto tiende a ser selectivo (Farinola et al., 2018), sin lugar a dudas la práctica del esquí deportivo se potencia como elitista. ${ }^{8}$ En términos concretos, menos de 300 menores de 18 años residentes de Bariloche acceden al pase de esquí para practicar el deporte a través de los clubes locales (CAPSA, 2018), es decir, menos del $3 \% 0 .{ }^{9}$ Dentro de ese grupo reducido, apenas una parte puede realizar la modalidad deportiva con todas las características de la dimensión que la define.

En comparación con otros tipos de prácticas, en Bariloche son muy pocas las personas que pueden realizar esquí de manera recreativa. El reducido porcentaje de residentes barilochenses que sí acceden a esquiar o a hacer snowboard centran su práctica en la dimensión recreativa. También existe un número muy menor de personas que realizan prácticas de esquí de travesía (modalidad de ascenso con pieles removibles) y de

8 La competencia deportiva de esquí requiere disponibilidad de amplios recursos. Demanda acceder a un equipamiento que es muy costoso, implica sostener entrenadores durante toda la temporada, asistir al calendario de la categoría para mantenerse rankeado y acceder a competencias internacionales en el hemisferio opuesto.

9 Esquiar se acota a tres meses en el año (julio-septiembre) y los días reales para hacerlo, descontados los de escolarización, tienden a ser unos 40 (CAPSA, 2018). 
esquí de fondo. Pero, aun agrupando todas las variantes, estas prácticas invernales de deslizamiento se muestran como minoritarias y restringidas a un período muy acotado del año.

\section{¿Intentos fallidos o exitosos?}

El proyecto de ciudad turística proyectado a partir de 1934 sentó bases sólidas que se sostuvieron y se reflejan en el recorrido material y simbólico de las prácticas de esquí. Sin soslayar la amplitud de prácticas y sentidos que tiene intrínsecamente una sociedad, como hecho social existe una conformación imaginaria colectiva que deviene estructurante en una identidad socialmente compartida que ubica al esquí como una práctica de elite.

Luego, los gobiernos nacionales, provinciales y locales no plasmaron en sus gestiones el hecho de desligar la orientación turística de la ciudad ni fueron intercediendo en la definición del acceso de sus habitantes al patrimonio regional, cultural y natural en el que vivían. Con su crecimiento muy ligado a las migraciones internas y externas, sumado al crecimiento desregulado y vinculado al desarrollo turístico nacional e internacional, se asentó la constitución de una ciudad turística para los visitantes (afianzada en el centro, la costa de lago Nahuel Huapi y los cerros Catedral y Otto), separada de otra ciudad de población residente al servicio de la primera. Dicho de otra manera, al ceder su regulación al mercado, fuertemente vinculado al turismo, fue esa lógica la que delineó el desarrollo de la ciudad, sus límites y posibilidades.

Hasta mediados de la década de 1980, las experiencias vinculadas a la práctica del esquí de sectores sociales medios o bajos tendieron a ser esporádicas, acotadas, mayormente desvinculadas de cualquier decisión del Estado Nacional, Provincial o Municipal, y quedaron en manos de particulares. A partir de la creación de la Escuela Municipal de Esquí, en 1984, fueron apareciendo variantes que, aunque erráticas o inconstantes, adquirieron mayor alcance con el crecimiento del proyecto de Esquí Escolar.

\section{De íconos, distinción y desafíos}

En la región andina de Río Negro, como un ícono de gran fuerza simbólica, se puede establecer una analogía entre el acceso al ambiente natural en general y las prácticas de esquí de sus habitantes en particular. La evidencia indica que, desde el inicio, el esquí resultó ser una práctica de unos pocos habitantes que poseían los bienes para realizarla, tiempo y habilidades adquiridas específicamente en viajes a Europa. 
Desde la intervención de Parques Nacionales, en 1934, el esquí pasó a convertirse en una insignia de distinción de las elites porteñas - y eventuales extranjeros - y una imagen de propaganda de la Suiza argentina. Con una gran inversión económica del Estado Nacional, se desplegó todo un aparato simbólico que modificó la idiosincrasia de la región, de sus modos de producción y de su proyección hacia el futuro: el turismo vinculado al ambiente natural se transformaría en la principal economía.

Este proceso hegemónico tuvo resistencias que se fueron evidenciando a partir de intervenciones que intentaron enseñar la práctica del esquí a personas normalmente excluidas. El hecho de facilitar la práctica del esquí a sectores sociales vulnerados o que nunca podrían hacerlo remite en gran parte a un posicionamiento político concreto y disruptivo. Las personas de sectores sociales bajos o medio-bajos no podrían nunca esquiar, a menos que mediara una situación fuera de lo naturalizado.

Quienes intervinieron para transformar esta práctica, que sostenía y acentuaba las diferencias sociales, se posicionaron en un espacio en el cual pensaron que sus acciones podrían modificar algunos aspectos de la realidad, como la accesibilidad del patrimonio regional (natural por su entorno y cultural por sus prácticas), y también proyectarlos a toda la población, apelando a modos democráticos más equitativos. La enseñanza del esquí para sectores vulnerados o excluidos resultaba una opción posible, a la vez que socialmente disruptiva.

En la construcción de un paisaje patagónico europeizado (Galafassi, 2019), de cerros nevados, chalés, pinos exóticos introducidos, inmigrantes del hemisferio norte enaltecidos y nativos ocultados, la práctica del esquí para sectores populares estaba fuera de lugar. Para Bariloche, el esquí es una práctica histórica, culturalmente valorada, estructuralmente posible y simbólica y normalmente excluyente.

Los modos de producción material y simbólico han contribuido a que, en el noroeste de la Patagonia, el acceso al ambiente natural y sus prácticas fuera algo excluyente del común de su población. Sin embargo, en oposición al proyecto centralista y aristocrático que tendió a instalar y promover las prácticas de esquí y el disfrute de los ambientes naturales para un sector social alto, se fue acrecentando y desarrollando una secuencia variada y circunstancial de propuestas que promovieron la incorporación del esquí y la apropiación del ambiente en los habitantes de Bariloche. Estas propuestas van sentando las bases de un nuevo imaginario que se construye de manera local, en un marco social más democrático e inclusivo. 


\section{Referencias bibliográficas}

Aisenstein A., y Scharagrodsky P. (2006). Tras las huellas de la educación física escolar argentina: Cuerpo género y pedagogía 1880-1950. Buenos Aires: Prometeo.

Arias Bucciarelli, M. (2009). La Patagonia argentina como territorio nacional: Perspectivas de análisis. Ponencia presentada en VIII Congreso de Historia Social y Política de la Patagonia Argentino-Chilena, Trevelin.

Arko, T. (2006). Ski deportivo en Argentina. Bariloche: Caleuche.

Bachmann, M. (2000). Historia del esquí en Bariloche. Bariloche: Esegé.

Bourdieu, P. (2002). La distinción: Criterios y bases sociales del gusto. México: Taurus.

Bustillo, E. (1968). El despertar de Bariloche: Una estrategia patagónica. Buenos Aires: Editorial y Librería Goncourt.

Catedral Alta Patagonia S.A. (2018). [Aspectos económicos y sociales de la concesión de cerro Catedral: Informe a la municipalidad presentados en la sesión pública de 16/02/2018]. Recuperado de http://concejobariloche.gov.ar/index.php/documentacion/1066-presentacio-n-16-02.

Chiappe, M., y Méndez, L. M. (2021). ¡A lo macho! Masculinidades hegemónicas en la región del Nahuel Huapi, 1930-1955. En P. Scharagrodsky (comp.), Hombres en movimiento: Deporte, cultura física y masculinidades en la Argentina 1880-1970 (pp. 297-319). Buenos Aires: Prometeo.

Chiocconi M., Chiappe M., y Podlubne, A. (2011). ¡Siempre listos por la Patria! Del scoutismo internacional al scoutismo nacional. En L. Méndez (dir.), Historias en movimiento: Cuerpo educación y tiempo libre en la norpatagonia 1884-1945 (pp. 181247). Rosario: Prohistoria.

Chiocconi, M. (2015). La trascendencia política del esquí: Una razón de Estado en la Argentina de la primera mitad del siglo XX. En L. Méndez y A. Podlubne (comps.), Tiempo de jugar, tiempo de aprender: Educación, museos y prácticas corporales en la Patagonia Norte, 1910-1955 (pp. 213-239). Buenos Aires: Prometeo.

Farinola, M., Tuñón I., Laíño F., Marchesich M., y Pérez Rodríguez M. (2018). Perfil socioeducativo y económico de deportistas adolescentes de élite argentinos. Retos Nuevas Tendencias en Educación Física, Deporte y Recreación, (34), 172-176.

Fuentes, R., y Núñez, P. (2007). 1. Sectores populares: Identidad cultural e historia en Bariloche. Bariloche: Núcleo Patagónico.

Fuentes, R., y Núñez, P. (2008). 2. Robles-Pilar 1: Identidad y lucha por la tierra en San Carlos de Bariloche. Bariloche: Núcleo Patagónico.

Galafassi, G. (2019). Paisaje y territorio en la construcción histórica de la región AndinoPatagónica. Terra: Nueva Etapa, 35(58).

Guevara, T., y Núñez, P. (2014). La ciudad en disputa: Desarrollo urbano y desarrollo económico en San Carlos de Bariloche. Diálogo Andino, (45), 153-167. 
Imágenes del Club Andino Bariloche años 30 - Esquiadores cerro Otto. (s.d.). [video]. Archivo General de la Nación, HB0931, Buenos Aires.

Instituto Nacional de Estadísticas y Censos. (1914). III Censo Nacional. Recuperado de: https://biblioteca.indec.gob.ar/bases/minde/1c1914t1.pdf.

Instituto Nacional de Estadísticas y Censos. (1947). IV Censo General. Recuperado de https://www.mininterior.gov.ar/poblacion/archivos_estadisticas/EvolucionPoblacionProvincias1914.pdf

Instituto Nacional de Estadísticas y Censos. (2010). Censo Nacional de Población, Hogares y Viviendas 2010. Recuperado de https://www.indec.gob.ar/indec/web/Nivel4-Tema-2-41-135.

Kirk, D. (2010). Physical education futures. Nueva York: Routledge.

Mársico, L. (2020). Currículums en disputa: La incorporación del esquí en la propuesta curricular de las escuelas públicas de nivel primario de San Carlos de Bariloche, Río Negro (1999-2013) (Tesis de maestría). Universidad Nacional de Quilmes, Bernal, Argentina. Recuperado de http://ridaa.unq.edu.ar/handle/20.500.11807/1906.

Matossian, B. (2015). Desigualdades socioterritoriales, fracturas urbanas y escala barrial en San Carlos de Bariloche: El rol de las Juntas Vecinales. Ponencia presentada en las XI Jornadas de Sociología: Coordenadas contemporáneas de la sociología. Tiempos, cuerpos, saberes.

Medina, V. (2017). Crecimiento urbano y desigualdad espacial: El rol del entorno natural en San Carlos de Bariloche. Bitácora Urbano-Territorial, 27(2), 101-108.

Municipalidad de San Carlos de Bariloche. (1984, 30 de agosto). Ordenanza 102-C-84: Escuela Municipal de Esquí - Concejo Municipal. Boletín Oficial RN, 2177.

Municipalidad de San Carlos de Bariloche. (2008). Relevamiento socio territorial: Informe general.

Municipalidad de San Carlos de Bariloche. (2011). Políticas, instrumentos y proyectos para el ordenamiento territorial de San Carlos de Bariloche: Plan de ordenamiento territorial.

Municipalidad de San Carlos de Bariloche. (2015). Primer esquema del plan estratégico e integral de desarrollo de San Carlos de Bariloche.

Municipalidad de San Carlos de Bariloche. (2021). Arribo de turistas y estadía media: Informaciones del período ene/2006 - mar/2020. Recuperado de www.bariloche.gov.ar/estadisticas_grafico.php?grafico=2.

Navarro Floria, P. (2008). El proceso de construcción social de la región del Nahuel Huapi en la práctica simbólica y material de Exequiel Bustillo (1934-1944). Pilquen: Sección Ciencias Sociales, 10(1), 1-14.

Navarro Floria, P., y Vejsbjerg, L. (2009). El proyecto turístico barilochense antes de Bustillo: Entre la prehistoria del Parque Nacional Nahuel Huapi y el desarrollo local. Estudios y Perspectivas en Turismo, 18(4), 414-433. 
Núñez, P. (2014). La región del Nahuel Huapi en el último siglo: Tensiones en un espacio de frontera. Pilquen: Sección Ciencias Sociales, 17(1), 5.

Núñez, P., y Vejsbjerg, L. (2010). El turismo, entre la actividad económica y el derecho social: El Parque Nacional Nahuel Huapi, Argentina, 1934-1955. Estudios y Perspectivas en Turismo, 19, 930-945.

Promoción Turística - Esquí - Puerto Blest década de 1940 (s.d.). [video]. Archivo General de la Nación, HB1395, Buenos Aires.

Ros \& Assoc. (1998). Plan estratégico y de competitividad turística de San Carlos de Bariloche: Documento de síntesis.

Scharagrodsky, P. (comp.). (2011). La invención del «homo gymnasticus»: Fragmentos históricos sobre la educación de los cuerpos en movimiento en Occidente. Buenos Aires: Prometeo Libros. 